CLINICAL STUDY

\title{
Fractionated stereotactic radiotherapy in patients with acromegaly: an interim single-centre audit
}

\author{
$\mathrm{S} \mathrm{Roug}^{1}$, А K Rasmussen ${ }^{1}$, M Juhler ${ }^{2}$, M Kosteljanetz ${ }^{2}$, L Poulsgaard ${ }^{2}$, H Heebøll ${ }^{3}$, H Roed ${ }^{4}$ and U Feldt-Rasmussen $^{1}$ \\ Departments of ${ }^{1}$ Medical Endocrinology PE 2132, ${ }^{2}$ Neurosurgery, ${ }^{3}$ Radiology, and ${ }^{4}$ Radiation Oncology, Rigshospitalet, National University Hospital, \\ Blegdamsvej 9, DK-2100 Copenhagen, Denmark
}

(Correspondence should be addressed to U Feldt-Rasmussen; Email: ufeldt@rh.dk)

\begin{abstract}
Aim: To evaluate the effect of fractionated stereotactic radiotherapy (FSRT) in acromegaly in a retrospective analysis.

Patients and methods: Thirty-four patients (17 females, median 43 years (range 30-74)) with acromegaly were treated with FSRT (conformal dynamic arcing, dose 54 Gy, 27-30 fractions) between January 1998 and April 2007. Of the 34 patients, 32 had undergone transsphenoidal adenotomy, and 28 were on medical therapy before FSRT. Patients on medical therapy continued this during and after the irradiation. The treatment was gradually decreased/withdrawn after careful assessment.

Results: Magnetic resonance scanning of the pituitary gland 34 months (median, range 11-95) after irradiation showed stable or reduced volume of the remaining tumour tissue in 31 of 34 patients (91\%). Seventeen patients $(50 \%)$ were biochemically controlled (normalised nadir GH during oral glucose tolerance test and IGF1 <+2 s.D.) 30 months after FSRT (median, range 6-60), and ten of them had true biochemical remission (off medical therapy) 30 months after FSRT (median, range 12-69). Of 28 patients with one or more functioning pituitary axes before irradiation, 8 (29\%) developed further deficit of one or two pituitary axes 48 months (median, range 6-102) after FSRT. Of 34 patients, 20 still required medical treatment for acromegaly at the end of this study, mainly those with a short follow-up period after irradiation.

Conclusion: The FSRT seems promising in terms of treatment of acromegaly. Longer follow-up is, however, needed to assess the overall efficacy and safety of FSRT for acromegaly.
\end{abstract}

European Journal of Endocrinology 162 685-694

\section{Introduction}

Acromegaly, GH hypersecretion, is associated with increased morbidity and mortality, and therefore treatment is needed $(1,2)$. Conventional treatment of GH-secreting pituitary adenoma is primarily transsphenoidal surgery where cure or adequate control can be expected in $>80 \%$ of cases when the tumour is within the sella (3). Besides the size and location of the tumour, the expertise of the surgeon is a determinant of the outcome of the surgery (4). Medical therapy, mostly as somatostatin analogues and/or GH receptor antagonist, is an alternative treatment option (4). Even though medical therapy is efficient in controlling acromegaly, radiotherapy (RT) may still have a role both on the hormonal level and on tumour mass control (3) in a subgroup of acromegalic patients. RT is mainly performed in patients with an inoperable tumour, unacceptable hormone level despite surgery and medical therapy, or progressive tumour growth $(4,5)$.

Stereotactic techniques have improved RT of acromegaly - mainly due to the high accuracy in the radiation delivery (6). Only stereotactic radiosurgery (SRS) has documented faster hormonal effect than conventional RT (3), but overall SRS and fractionated stereotactic RT (FSRT) result in the same local control (6). FSRT, though, is recommended when the tumour is close to critical structures $(6,7)$.

FSRT is being used as a treatment modality for acromegaly at Rigshospitalet, Copenhagen, since January 1998. The aim of this study was to evaluate, in a retrospective analysis of a consecutive cohort of patients with FSRT-treated acromegaly, the effect of FSRT on control of GH overproduction/insulin-like growth factor 1 (IGF1) with (controlled acromegaly) or without medical therapy (true remission), magnetic resonance (MR) scan control of tumour size, persistent medical treatment with somatostatin analogues (Sandostatin LAR, Novartis, Basel, Switzerland) and/or a GH receptor antagonist (Somavert, Pfizer, New York, USA), and function of other pituitary axes.

\section{Patients and methods}

Thirty-four consecutive patients (17 females, median 43 years (range 30-74)) with GH-secreting pituitary 
adenomas treated with FSRT at Rigshospitalet, Copenhagen, between January 1998 and April 2007 entered the study. The indication for irradiation was persistent acromegaly after surgery and/or medical treatment, or intolerance to the medical treatment. No patients were excluded from the study, and no patient was lost during follow-up.

Time between diagnosis of acromegaly and FSRT was 35 months (median, range 5-346). Of the 34 patients, 31 (91\%) had undergone primary transsphenoidal adenotomy. Nine patients had undergone surgery more than once. Time between operation and FSRT was median 16 months (range 4-345). Three patients were treated primarily with FSRT since the location of the adenoma made FSRT more suitable than surgery. One of these patients, however, had to undergo surgery 37 months after FSRT due to lack of response and intolerance to both somatostatin analogue and $\mathrm{GH}$ receptor antagonist therapy. The other two patients were not biochemically controlled despite treatment with somatostatin (Tables 1 and 2).

The patients were treated according to ethics described in the Helsinki II declaration, and the study has been listed in ClinicalTrials.gov (ID NCT00934271). There is no requirement in Denmark for informed consent and approval from the ethical committee of an audit.

\section{Biochemical acromegaly status (IGF1 and nadir GH)}

The criteria for biochemical success were nadir $\mathrm{GH}$ $<2.6 \mathrm{mU} / \mathrm{l}$ by oral glucose tolerance test and IGF1 below +2 s.D. of gender- and age-matched normal healthy individuals. Biochemical control was defined as above biochemical success irrespective of medical therapy status. True biochemical remission was defined as biochemical control as defined above without adjunctive medical therapy. IGF1 and nadir GH were measured every 6 months after FSRT.

Two patients were biochemically controlled on medical therapy pre FSRT, and were treated with FSRT due to serious side effects of medical therapy (Sandostatin LAR $20 \mathrm{mg}$ i.m./month) or to severe symptoms of headache and impaired vision despite medical therapy (Sandostatin LAR $30 \mathrm{mg}$ i.m./month and Somavert $15 \mathrm{mg}$ s.c./day), which were considered to be local symptoms from the remaining tumour tissue.

Ten patients had discordant measurements of IGF1 and nadir GH prior to FSRT, two of them with elevated IGF1 and normal nadir GH, and eight with elevated nadir GH and normal IGF1 (Table 2).

\section{MR scan}

By an individual estimate, pituitary MR scan was done every 12-24 months, and was evaluated by the same radiologist to avoid interpersonal variation. Tumour volume was evaluated in three diameters: anterior-posterior, cranial-caudal and side-side. Median tumour volume before FSRT was $4.5 \mathrm{~cm}^{3}$ (range 0.9-38.6). Tumour tissue that had a growth $<2 \mathrm{~mm}$ was considered stable tumour tissue. Tumour control was defined as regression or stable (remaining) tumour tissue. The expansion of the tumour was divided into intra- and extrasellar extension (Table 3).

\section{Medical therapy}

Of 34 patients, $28(82 \%)$ had been on medical therapy prior to FSRT for 21.5 months (median, range 4-126). The medically treated patients had been on either somatostatin analogue (Sandostatin LAR) or GH receptor antagonist (Somavert), or a combination of both for variable periods of time from 4 to 126 months (Tables 1 and 2).

No patients were advised to stop medical therapy during the period of FSRT. The need for continued medical therapy was estimated individually, and all patients were assessed at least every 6 months after FSRT based on clinical side effects and biochemical acromegaly status.

\section{Pituitary function}

ACTH deficiency was diagnosed if basal or stimulated (synacthen or insulin tolerance test) cortisol levels did not reach $500 \mathrm{nmol} / \mathrm{l}$. All blood tests were performed in a fasting state between 0900 and $1100 \mathrm{~h} \mathrm{(8).} \mathrm{TSH}$ deficiency was defined when free thyroxine $\left(\mathrm{FT}_{4}\right)$ was low (below or in the low normal range) with an inappropriately low TSH. LH/FSH deficiency was defined when testosterone or oestradiol concentrations were low combined with inappropriately low FSH and LH, and/or the FSH and LH response to GnRH was less than twofold. Panhypopituitarism was defined as deficiencies of all three axes. Replacement therapy was initiated when clinical signs and/or biochemistry indicated the need. Of the 34 patients, $30(88 \%)$ had hypopituitarism before FSRT, 6 of them with panhypopituitarism (Table 4). Time intervals for evaluation of the pituitary function were estimated individually, and the evaluation was performed at least every 6 months in all 34 patients.

Twenty-two patients $(65 \%)$ had received hormone replacement therapy prior to FSRT for a median of 22 months (range 2-165). Three patients were substituted on three axes ( $\mathrm{L}_{-} \mathrm{T}_{4}$, hydrocortisone and testosterone), and ten patients on two axes ( $\mathrm{L}_{-} \mathrm{T}_{4}$ and hydrocortisone), while nine patients were substituted on only one axis (seven with hydrocortisone and two with testosterone). Two women were on oral contraceptives.

\section{Assays}

GH was analysed by a fluoroimmunometric assay (AutoDelfia, Perkin-Elmer, Wellesley, MA, USA) calibrated against the International RP 80/505 


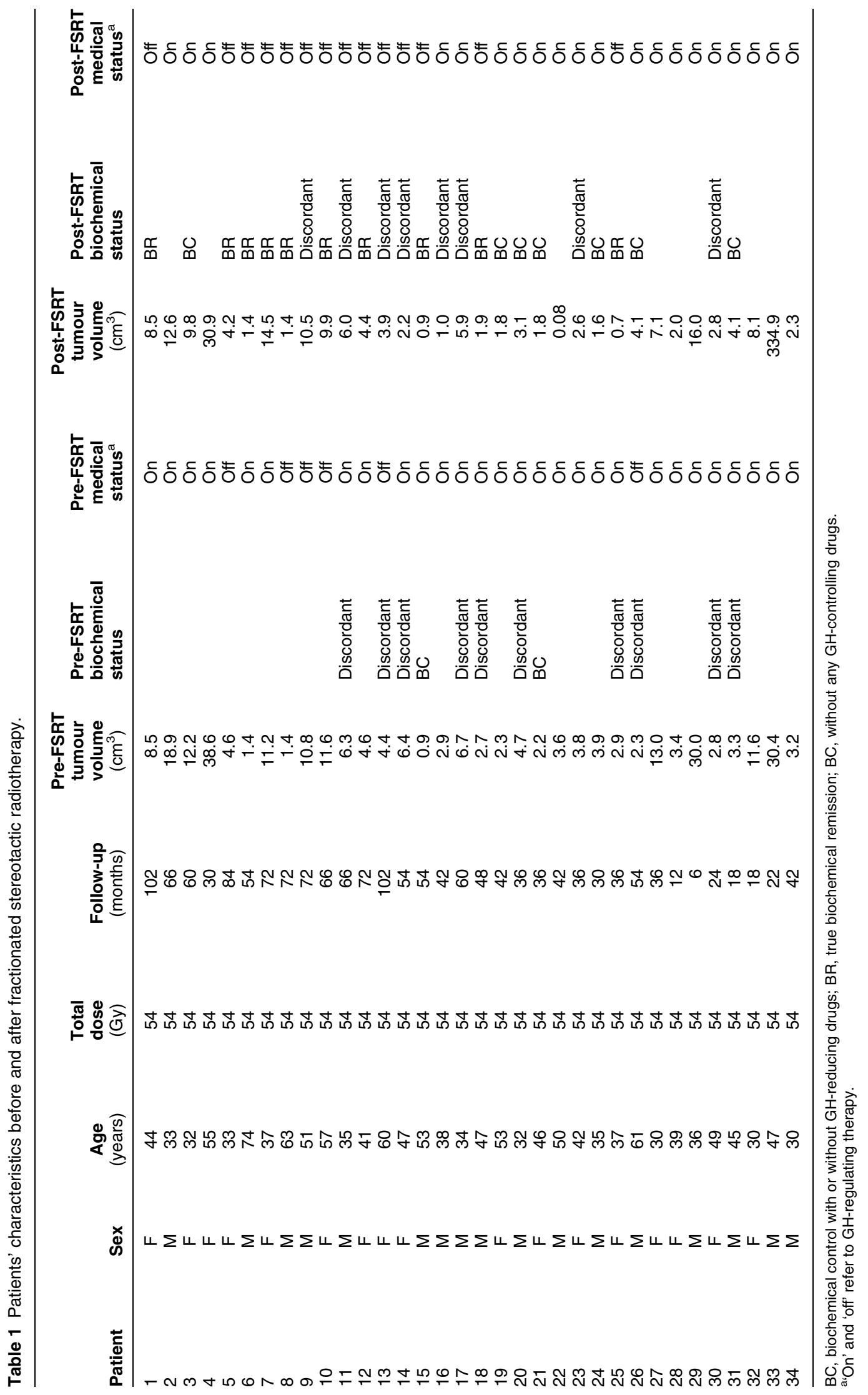




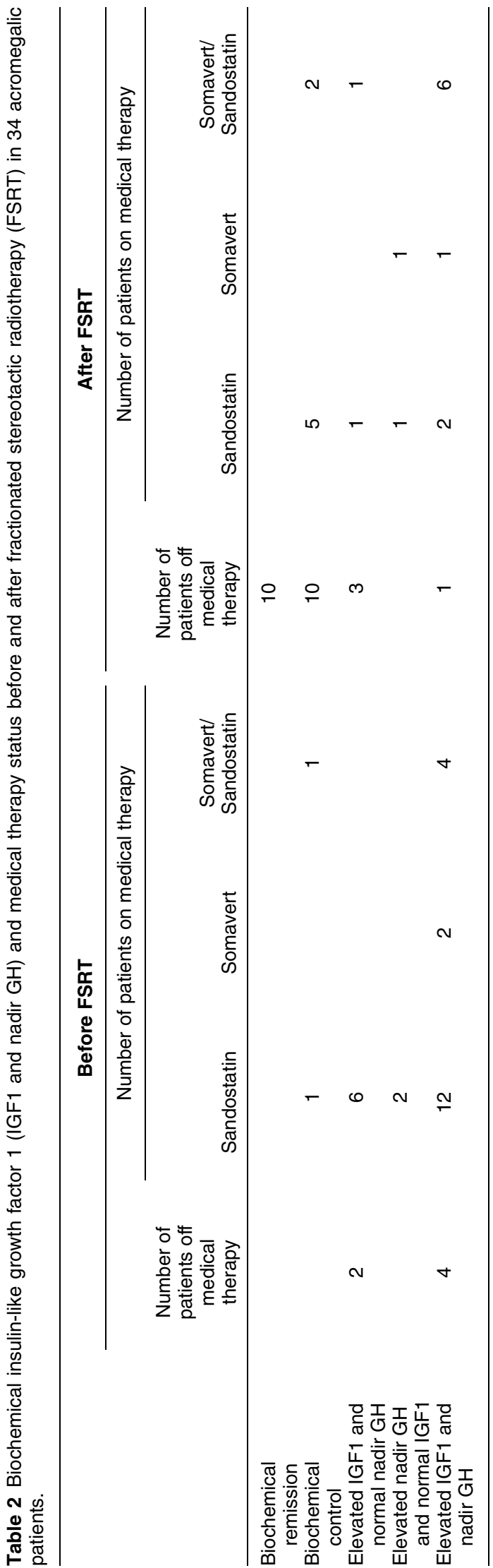

( $1 \mathrm{mg}=2.6 \mathrm{IU})$. Intra- and interassay coefficients of variations $(\mathrm{CV})$ were $2.2-5.1$ and $1.4-2.5 \%$ respectively.

IGF1 was initially analysed by a RIA with intra- and interassay CV of 3.1 and 10\% respectively. After January 2008, IGF1 was analysed by a solid-phase enzyme-labelled chemiluminescent immunometric assay with intra- and interassay $\mathrm{CV}$ of $2.3-3.9$ and $3.7-8.1 \%$ respectively. Calculations of age- and gender-adjusted SDS for IGF1 (IGF1-SDS) were published previously together with the reference data used in the calculation (9).

\section{Fractionated stereotactic radiotherapy}

The patients were treated with accelerator-based irradiation in normally conformal dynamic arcs. An individual mask of the head was made during the planning process, and was used during all the treatments. The mask was fixed to the board during the radiation, thereby creating immobilisation. A fusion of MR and computed tomography (CT) scans was used as a visual treatment plan, whereby the gross target volume (GTV) was estimated. A 3-mm margin was added to account for set-up uncertainties and penumbra. All dose planning was done in BrainLab (Munich, Germany) dose planning system. All patients were treated with $54 \mathrm{~Gy}$ in 27-30 fractions prescribed to the periphery of the GTV during 5.5-6 weeks.

\section{Statistical analysis}

All calculations were made in GraphPad Software. Fisher's exact test and paired $t$-test were used, and a level of 0.05 was considered significant.

\section{Results}

\section{Biochemical acromegaly status (IGF1 and nadir GH)}

Biochemical follow-up was available in all 34 patients 45 months after FSRT (median, range 6-102; Tables 1 and 2). Seventeen patients were biochemically controlled $(50 \%) 30$ months after FSRT (median, range 6-60), and none of the 17 patients had biochemical relapse during the subsequent follow-up period of 18 months (median, range 6-72). Of the 17 patients, ten even had true biochemical remission (biochemical control off medical therapy) 30 months after FSRT (median, range 12-69). Seven patients withdrew medical therapy after FSRT, and three patients, who tried medical therapy before FSRT, were intolerant to both drugs. None of the ten patients in true remission had biochemical relapse during the subsequent follow-up period of 26 months (median, range 6-72).

Of the 17 biochemically controlled patients, 2 were biochemically controlled already before FSRT, and both remained in this state during follow-up. One stopped 
Table 3 Tumour expansion in 34 acromegalic patients before and after fractionated stereotactic radiotherapy (FSRT).

\begin{tabular}{lcr}
\hline & Before FSRT & After FSRT \\
\hline Intrasellar extension total & $5(15 \%)$ & $6(18 \%)$ \\
Extrasellar extension (E) total & $29(85 \%)$ & $28(82 \%)$ \\
E - suprasellar & $12(35 \%)$ & $10(29 \%)$ \\
E - infrasellar & $13(38 \%)$ & $15(44 \%)$ \\
E - anterior & $1(3 \%)$ & $0(0 \%)$ \\
E - posterior & $3(9 \%)$ & $4(12 \%)$ \\
E - parasellar bilateral & $5(15 \%)$ & $5(15 \%)$ \\
E - parasellar dex & $11(32 \%)$ & $11(32 \%)$ \\
E - parasellar sin & $12(35 \%)$ & $11(32 \%)$ \\
\hline
\end{tabular}

medical therapy, and was therefore also considered in true biochemical remission 27 months after FSRT and for the following 27 months, while the other patient remained on medical therapy (Sandostatin LAR and Somavert) during the follow-up period of 36 months.

IGF1 (SDS) was +0.5 (median, range $-1.85 \pm 1.8$ ) in the 17 biochemically controlled patients 54 months after FSRT (median, range 18-102), and it was + 0.64 (median, range $-1.77 \pm 1.8$ ) in the ten patients in true biochemical remission 69 months after FSRT (median, range 36-102).

True biochemical remission rate was 50\% (3 of 6 patients) in the group of patients off medical therapy during FSRT compared with $25 \%$ ( 7 of 28 patients) in the group of patients treated with somatostatin analogues and/or GH receptor antagonist.

The biochemical control rate after 1 year was $24 \%$ ( $n=8 / 34$; with seven of the eight on medical therapy), after 3 years was $38 \%(n=10 / 26$; with six of the ten on medical therapy) and after 5 years was $64 \%(n=7 / 11$; with three of the seven on medical therapy).

Eight patients $(24 \%)$ had at the last follow-up measurement discordant IGF1 and nadir GH concentrations, three of whom had elevated nadir GH with normal IGF1, and five had elevated IGF1 with normal nadir GH (one of the five patients had FSRT before surgery). Nine patients $(26 \%)$ did not normalise nadir GH or normalise IGF1 during follow-up (two of these nine patients were treated primarily with FSRT combined with medical therapy).

IGF1 (SDS) in the 17 patients who did not normalise both IGF1 and nadir GH was +3.12 (median, range

Table 4 Pituitary function in 34 acromegalic patients before and after fractionated stereotactic radiotherapy (FSRT) in four categories: normal function and deficiency of 1,2 and 3 axes respectively.

\begin{tabular}{lrr}
\hline & Before FSRT & After FSRT \\
\hline Normal pituitary function & $4(12 \%)$ & $1(3 \%)$ \\
One axis deficient & $19(56 \%)$ & $15(47 \%)$ \\
Two axes deficient & $5(15 \%)$ & $10(29 \%)$ \\
Three axes deficient & $6(17 \%)$ & $8(21 \%)$ \\
$\quad$ (panhypopituitarism) & & \\
\hline
\end{tabular}

$-0.77 \pm 5.5) 42$ months after FSRT (median, range 6-102).

Mean nadir GH $(n=34)$ dropped from $12.8 \mathrm{mU} / \mathrm{l}$ (range 1.3-149) pre FSRT to $6.4 \mathrm{mU} / \mathrm{l}$ (range 0.1-96.5) 45 months (median, range 6-102, 95\% confidence interval (CI) 2.6-10.2) after FSRT $(P=0.0016)$, while mean IGF1 $(n=34)$ decreased from $510 \mu \mathrm{g} / \mathrm{l}$ (range 193-974) pre FSRT to $261 \mu \mathrm{g} / \mathrm{l}$ (range 97-605) 45 months (median, range 6-102, 95\% CI 190-309) after FSRT (P0.0001).

\section{MR scan}

Radiographic follow-up was available in all 34 patients 34 months (median, range 11-95) after FSRT. Thirty-one patients $(91 \%)$ had tumour control, 17 of them $(55 \%)$ with regression of the tumour tissue at a median followup time of 32 months (range 12-95; 1 of the 17 patients was treated primarily with FSRT), while 14 patients (45\%) had stable tumour tissue after a median of 43 months (range 11-70; 2 of the 14 patients had primary FSRT and 1 of the 2 patients was operated afterwards).

The remaining three patients $(9 \%)$ had progression of the tumour tissue in median 23 months (range 20-27) after FSRT. One of them was known with malignant metastases from the pituitary tumour, and progression was found in all three diameters. The remaining two patients had progression of both the cranial-caudal and side-side diameters.

Median tumour volume after FSRT was $4.0 \mathrm{~cm}^{3}$ (range 0.08-334.9). Without the one patient known with malignant pituitary tumour, median tumour volume was $3.9 \mathrm{~cm}^{3}$ (range 0.08-30.9) after FSRT. There was no statistical difference between tumour volume before and after FSRT $(P=0.4)$.

No statistical difference was found in tumour volume expansion between the numbers of patients with intrasellar and extrasellar tumour extension before and after FSRT ( $P=1$; Table 3$)$. There was no evidence of secondary tumour development, cerebral necrosis or vascular changes during the follow-up on MR scan.

\section{Medical therapy}

The medical therapy was withdrawn in 9 of 28 patients (32\%) after 33 months (median, range 23-72), and 7 of them remained biochemically controlled and therefore were considered in true biochemical remission. Two patients started the medical treatment after FSRT, one patient for a period of 66 months with a discordant biochemical value subsequent to withdrawal of the medication, and one patient was still on medical therapy at the end of this study, but had normal levels of IGF1 and nadir GH and was considered biochemically controlled.

Thus, 20 patients $(59 \%)$ were still on medical treatment at the end of this study compared with 28 patients ( $82 \%$ ) before FSRT. Follow-up time for these 20 patients was 36 months (median, range 6-66; Tables 1 and 2). 


\section{Pituitary function}

Follow-up time for the 34 patients was 45 months (median, range 6-102). Three of four patients $(75 \%)$ with completely normal function of the three pituitary axes before FSRT developed deficiency of two axes 27 months after FSRT (median, range 12-42). Of 24 patients with hypopituitarism before FSRT, 5 (21\%) experienced worsening of the pituitary function by new deficiencies 30 months (median, range 12-42) after FSRT, while 8 of 34 patients $(21 \%)$ had panhypopituitarism at the end of the study (Table 4).

New hormone replacement therapy was initiated in 16 patients 27.5 months (median, range $2-70$ ) after FSRT, 7 of whom (44\%) had to replace an additional axis 26 months (median, range 8-53) after FSRT.

No significant difference was found in the incidence of hormone deficits at the latest follow-up between the group of patients with a normal pituitary function prior to FSRT and the group of patients with hypopituitarism (deficits less than three axes) prior to FSRT $(P=0.35)$.

\section{Mortality}

One patient (3\%) died 22 months after FSRT due to metastatic GH-producing pituitary tumour. The tumour had an aggressive invasive behaviour before FSRT.

\section{Discussion}

The traditional algorithm for acromegaly advocates surgery and medical therapy as the first-line therapy, but between 10 and $30 \%(3,5)$ of patients with acromegaly still need additional treatment such as RT. Most publications on RT have reported results on fractionated conventional RT (10-12), and FSRT can therefore still be considered a new RT modality, which is also partly the reason why few studies have evaluated the role of FSRT in patients with acromegaly.

In the present study, 34 consecutive and prospective patients with acromegaly were evaluated retrospectively after treatment with FSRT between January 1998 and April 2007. The study was a single-centre audit, and as all patients irrespective of prior treatment and tumour size and growth were included, the patient population was very mixed. Seventeen patients were biochemically controlled and 15 of these 17 patients $(88 \%)$ also had tumour control. In total, ten patients were in true biochemical remission 30 months after FSRT (median, range 12-72). Tumour control was achieved in 31 of 34 patients $(91 \%)$ after a median of 34 months (range 11-95), and 17 of the tumours (55\%) even regressed.

It is difficult to compare the present study to existing papers when it comes to tumour control in acromegalic patients after FSRT, since most papers evaluated the www.eje-online.org

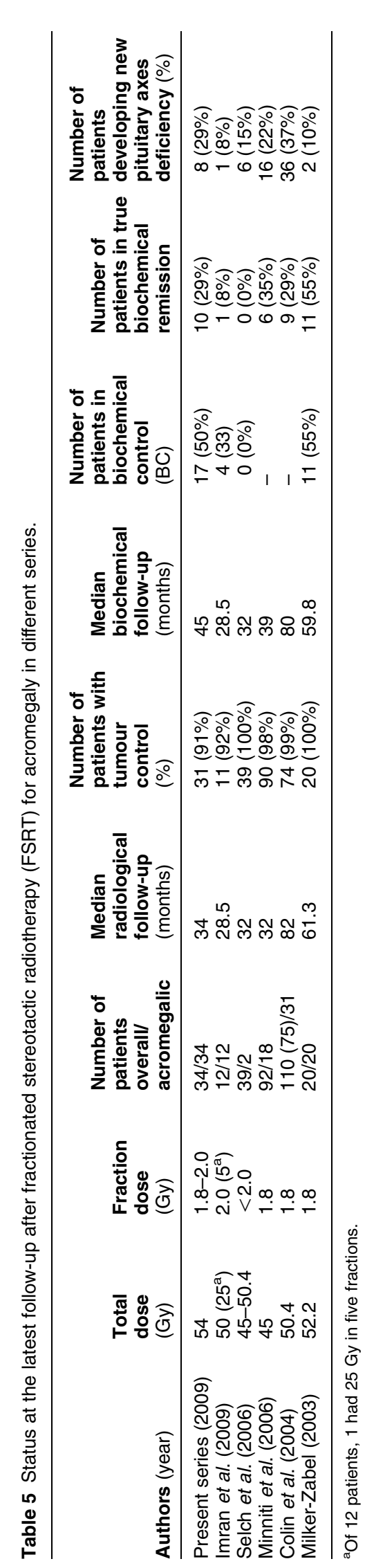


efficacy on a mixture of pituitary tumours, not confined to GH-producing adenomas (13-15), and the patients with acromegaly were difficult to identify from the other groups. Also, the criteria for biochemical success were not clear in some papers $(13,15)$. The present study thus brings new data on efficacy of both hormonal and radiological controls of acromegaly after FSRT, and also has the highest number of patients in which FSRT on acromegaly was evaluated $(n=34)(13-17)$.

Longer follow-up is expected to strengthen results in regard to control of the remaining tumour mass, since the time of follow-up for the radiological evaluation was limited to 34 months (median, range 11-95), and the effect of RT is known to develop years later (3) (Table 5). One of the three patients who had progression of the tumour mass died of metastases from the malignant tumour, which is a very rare occurrence (18). However, no secondary tumours, cerebral necrosis or vascular changes were identified in any of the other patients after FSRT.

Seventeen of our patients $(50 \%)$ were biochemically controlled by both IGF1 and nadir GH, and eight patients (24\%) had discordant values at the end. Biochemical control rate after 1 year was $24 \%, 3$ years was $38 \%$ and 5 years was $64 \%$. Of the 17 biochemically controlled patients, ten were considered to be in true biochemical remission since they were off medical therapy. Acceptable concentrations of both GH/nadir GH and IGF1 are important goals in the treatment of acromegaly in order to reduce morbidity and mortality (19), which is why we chose to include both IGF1 and nadir GH in the criteria for biochemical success in this study, even though normalisation of IGF1 with patients off medical therapy is often used as the sole criterion for true biochemical remission $(20,21)$.

Discordance between normalisation of the biochemical acromegaly status in regard to IGF1 and $\mathrm{GH} /$ nadir GH can be useful in the treatment and followup strategies, since elevated IGF1 alone seems to have a different prognosis compared with elevated $\mathrm{GH} /$ nadir GH alone $(22,23)$. Patients with elevated IGF1 and normal $\mathrm{GH} /$ nadir $\mathrm{GH}$ are at risk of having persisting clinical manifestations of acromegaly and can therefore indeed benefit from the treatment with a GH receptor antagonist $(24,25)$, while patients with elevated $\mathrm{GH} /$ nadir $\mathrm{GH}$ and normal IGF1 are at a risk of relapse of the tumour $(22,23)$. The origin of discordance is likely to be that IGF1 and GH/nadir GH seem to represent two different aspects of acromegaly (26), and RT in general reduces rather than cures the hypersecretion of GH $(3,24)$. Discordance is seen in up to $27 \%$ of acromegalic patients (22). This makes the discussion about criteria for cure relevant, and probably also substantiates a need for age-related limits in regard to $\mathrm{GH} /$ nadir $\mathrm{GH}$ and consensus regarding methods of analysis (27-29).

In general, we found results that were comparable to those of the existing papers on efficacy (13-17)

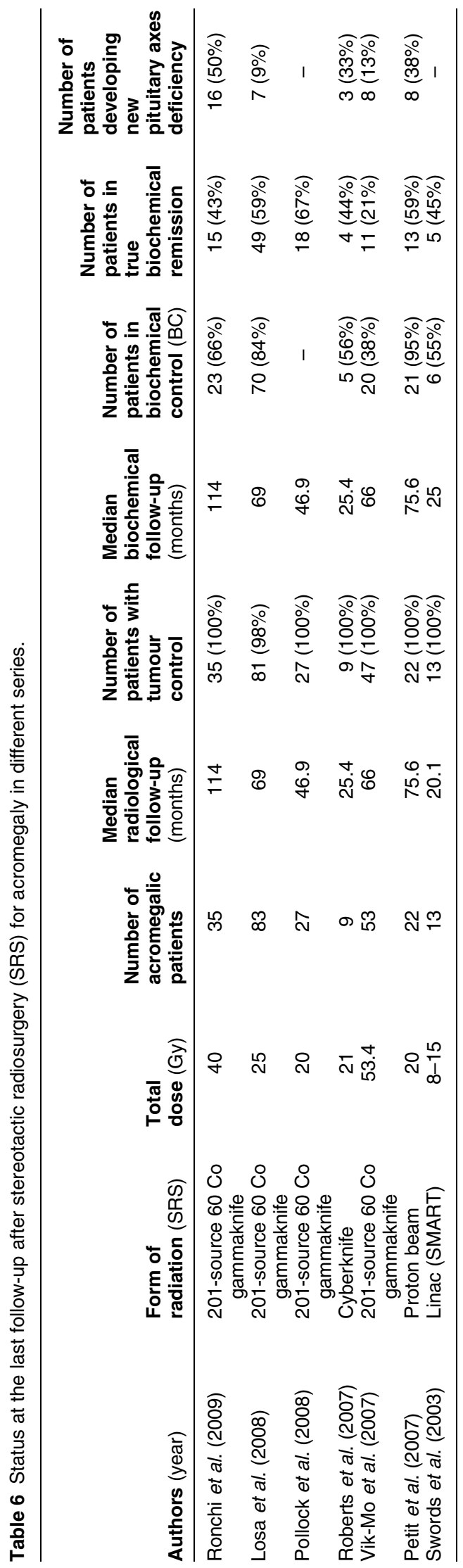

www.eje-online.org 
(Table 5). However, the present study has added knowledge about biochemical discordance between IGF1 and $\mathrm{GH} /$ nadir $\mathrm{GH}$, which was not evaluated in most existing papers regarding acromegalic patients treated with FSRT, probably because the acromegalic patients were a subgroup of patients with various pituitary tumours (13-15).

Comparison between patients treated with FSRT and SRS is complicated since allocation to the different stereotactic treatment modalities is different based on tumour size, shape and location $(6,7,30,31)$. By combining the high radiation precision known from SRS and the radiobiological advantages of fractionated radiation, FSRT is preferred over SRS when the target is close to a critical structure as, for example, the optic pathway $(13,32)$. Also, FSRT is performed mostly using a dental or head mask, thereby avoiding the invasive pins used during SRS (the Cyberknife is an exception) (6). However, SRS is more convenient for patients than FSRT, requiring 1 day versus 6 weeks of treatment (33).

Most series regarding FSRT to acromegalic patients have a follow-up time shorter than those for SRS (13-17, 20, 30, 32, 34-37) (Tables 5 and 6), which complicates the comparison since the effect of FSRT is known to appear later than the effect of SRS (6). However, the biochemical remission after SRS seems to vary between 21 and $67 \%$ (follow-up median 66 months, range 25-114) compared with 0 and 55\% after FSRT (follow-up median 42 months, range 28.559.8) (13-17, 20, 30, 32, 34-37) (Tables 5 and 6), but the criteria for biochemical remission in both the SRS and FSRT series were not identical. When allocated to the different stereotactic radiation methods by taking the limitations and advantages into consideration, the efficacy seemed equal (Tables 5 and 6).

Hypopituitarism is a serious side effect of RT of the pituitary gland in general $(4,24)$, and the incidence of new anterior pituitary hormonal deficiencies varies from $0 \%$ in the first years to $66 \%$ after a median follow-up of up to 17 years (38). Due to a slowly progressing effect of radiation on the pituitary gland, the highest incidence of hypopituitarism has been reported in the series of patients with the longest follow-up $(38$, 39). Risk of deficiency, though, seems maximal during the first 96 months after radiation (40). Evaluation of post-radiation hypopituitarism is difficult because of confounding factors such as previous surgery and/or radiation and differences in radiation of the tumour margin (38). The radiation dose in general, and to the pituitary stalk and the hypothalamus in particular, increased the incidence of hypopituitarism (6). This could be the reason why SRS of the pituitary gland compared with FSRT seemed to have a lower incidence of newly onset hypopituitarism (Tables 5 and 6). However, future studies are needed to clarify this. Also, deficiency prior to radiation has been reported to increase the vulnerability of the pituitary gland to radiation, and can therefore by itself increase the incidence (39). Yet, no statistical difference was found between the incidence of hypopituitarism in the patients with normal function of the pituitary gland and those with hormonal deficiencies prior to FSRT in this study.

The higher radiation precision by stereotactic techniques has not been proven to improve the cumulative incidence in long-term follow-up studies compared with conventional RT (38). Hypopituitarism is, like acromegaly, combined with increased mortality (41), which is an important reason for evaluating this side effect during the development of new radiation techniques. In the present series, $29 \%$ of the patients had a new deficit of one or two axes 48 months (median, range 6-102) after FSRT. Sixteen patients had hormone replacement therapy after FSRT, seven of whom (44\%) had to replace two axes. Compared with existing papers, we chose a high total radiation dose, although the fraction doses appeared alike (Table 5). Therefore, the low incidence of hypopituitarism $(n=8)$ is very interesting, and could indicate a high precision of radiation (6) in the present series.

Despite some indications that somatostatin analogues and the $\mathrm{GH}$ receptor antagonist could be radioprotective $(39,42)$, we chose to continue medical therapy during the period of radiation in order to limit the time of seriously elevated GH/IGF1. The present study has thus demonstrated that an acceptable treatment outcome can be achieved with FSRT, while the patients were still on medication, which is also in keeping with a demonstrated lack of significant difference between patients treated or not treated with somatostatin analogues (40). Current recommendations, however, seem to advise withholding medication during the radiation (6), and evaluation of biochemical measurements pre radiation without medical therapy seems to be a predictive factor of remission (40). However, only a prospective randomised study with sufficient power can clarify this issue.

In conclusion, FSRT may be used in acromegalic patients who have persistent or recurrent tumour growth or who are resistant/intolerant to medical therapy. Longer follow-up is, however, needed to assess the long-term efficacy and safety of FSRT for acromegaly. Since most of the patients had pituitary insufficiency before FSRT already, the main issue for follow-up that should be focused upon is the other long-term effects of irradiation, such as impaired cognition, morbidity and mortality, although the fertility issue should also be taken into account.

\section{Declaration of interest}

The authors declare that there is no conflict of interest that could be perceived as prejudicing the impartiality of the research reported. 


\section{Funding}

The study has been supported by a grant from Arvid Nilsson's Foundation.

\section{References}

1 Ben-Shlomo A \& Melmed S. Acromegaly. Endocrinology and Metabolism Clinics of North America 200837 101-122.

2 Holdaway IM. Excess mortality in acromegaly. Hormone Research 200768 166-172.

3 Monson JP. Is there still a role for radiotherapy in acromegaly? Neuroendocrinology 200683 269-273.

4 Freda PU. How effective are current therapies for acromegaly? Growth Hormone \& IGF Research 200313 144-151.

5 Castinetti F, Morange I, Dufour H, Regis J \& Brue T. Radiotherapy and radiosurgery in acromegaly. Pituitary 200912 3-10.

6 Shih HA \& Loeffler JS. Radiation therapy in acromegaly. Reviews in Endocrine \& Metabolic Disorders 20089 59-65.

7 Snead FE, Amdur RJ, Morris CG \& Mendelhall WM. Long-term outcomes of radiotherapy for pituitary adenomas. International Journal of Radiation Oncology, Biology, Physics 200871 994-998.

8 Klose M, Lange M, Kosteljanetz M, Poulsgaard L \& FeldtRasmussen U. Adrenocortical insufficiency after pituitary surgery: an audit of the reliability of the conventional short synacthen test. Clinical Endocrinology 200563 499-505.

9 Juul A, Bang P, Hertel NT, Main K, Dalgaard P, Jorgensen K, Muller J, Hall K \& Skakkebaek NE. Serum insulin-like growth factor-I in 1030 healthy children, adolescents and adults: relation to age, sex, stage of puberty, testicular size and body mass index. Journal of Clinical Endocrinology and Metabolism 1994 78 744-752.

10 Minniti G, Jaffrain-Rea M-L, Osti M, Esposito V, Santoro A, Solda F, Gargiulo P, Tamburrano G \& Enrici RM. The long-term efficacy of conventional radiotherapy in patients with $\mathrm{GH}$-secreting pituitary adenomas. Clinical Endocrinology 200562 210-216.

11 Jenkins PJ, Bates P, Carson MN, Stewart PM \& Wass JA. Conventional pituitary irradiation is effective in lowering serum growth hormone and insulin-like growth factor-I in patients with acromegaly. Journal of Clinical Endocrinology and Metabolism 2006 91 1239-1245.

12 Gutt B, Hatzach C, Morrison K, Pöllinger B \& Schopohl J. Conventional pituitary irradiation is effective in normalising plasma IGF-I in patients with acromegaly. European Journal of Endocrinology 2001144 109-116.

13 Selch MT, Gorgulho A, Lee SP, Mattozo C, Solberg TD, Agazaryan N \& DeSalles AAF. Stereotactic radiotherapy for the treatment of pituitary adenomas. Minimally Invasive Neurosurgery 200649 150-155.

14 Minniti G, Traish D, Ashley S, Gonsalves A \& Brada M. Fractionated stereo tactic conformal radiotherapy for secreting and nonsecreting pituitary adenomas. Clinical Endocrinology 2006 $64542-548$.

15 Colin P, Jovenin N, Delemer B, Caron J, Grulet H, Hecart AC, Lukas C, Bazin A, Bernard MH, Scherpereel B, Peruzzi P, Nakib I, Redon C \& Rousseaux P. Treatment of pituitary adenomas by fractionated stereo tactic radiotherapy: a prospective study of 110 patients. International Journal of Radiation Oncology, Biology, Physics 200562 333-341.

16 Milker-Zabel S, Zabel A, Huber P, Schlegel W, Wannenmacher M \& Debus J. Stereotactic conformal radiotherapy in patients with growth hormone-secreting pituitary adenoma. International Journal of Radiation Oncology, Biology, Physics 200459 1088-1096.

17 Imran SA, Fleetwood IG, O'Connell CM, Ransom T, Mulroy LA, Ur E \& Clarke DB. Outcome of stereotactic radiotherapy for patients with uncontrolled acromegaly. Canadian Journal of Neurological Sciences $200936468-474$.

18 Horvath E \& Kovacs K. Pathology of acromegaly. Neuroendocrinology 200683 161-165.
19 Colao A, Auriemma RS, Pivonello R, Galdiero M \& Lombardi G. Medical consequences of acromegaly: what are the effects of biochemical control? Reviews in Endocrine \& Metabolic Disorders $2008921-31$.

20 Roberts BK, Ouyang DL, Lad SP, Chang SD, Harch GR IV, Adler JR Jr, Soltys SG, Gibbs IC \& Katznelson L. Efficacy and safety of CyberKnife radiosurgery for acromegaly. Pituitary 2007 10 19-25.

21 Mullan K, Sanabria C, Abram P, McConnell M, Courtney H, Hunter S, McCance D, Leslie H, Sheridan B \& Atkinson B. Long term effect of external pituitary irradiation on IGF1 levels in patients with acromegaly free of adjunctive treatment. European Journal of Endocrinology 2009161 547-551.

22 Brooke AM \& Drake WM. Serum IGF-I in the diagnosis and monitoring of acromegaly. Pituitary 200710 173-179.

23 Clemmons DR. Clinical utility of measurements of insulin-like growth factor 1. Nature Clinical Practice. Endocrinology $\mathcal{E}$ Metabolism 20062 436-446.

24 Barkan AL. Radiotherapy in acromegaly: the argument against. Clinical Endocrinology 200358 132-135.

25 Hodish I \& Barkan A. Long-term effects of pegvisomant in patients with acromegaly. Nature Clinical Practice. Endocrinology $\mathcal{E}$ Metabolism 20084 324-332.

26 Tzanela M. Dynamic tests and basal values for defining active acromegaly. Neuroendocrinology 200683 200-204.

27 Jørgensen JO, Feldt-Rasmussen UF, Andersen M, Kristensen LØ, Laurberg P \& Weeke J. Acromegaly: new principles for treatment. Ugeskrift for Laeger $2007 \mathbf{1 6 9}$ 905-906.

28 Colao A, Pivonello R, Cavallo LM, Gaccione M, Auriemma RS, Espositot F, Cappabianca P \& Lombardi G. Age changes the diagnostic accuracy of mean profile and nadir growth hormone levels after oral glucose in postoperative patients with acromegaly Clinical Endocrinology 200665 250-256.

29 Espinosa-de-los-Monteros AL, Mercado M, Sosa E, Lizama O, Guinto G, Lopez-Felix B, Garcia O, Hernández I, Ovalle A \& Mendoza V. Changing patterns of insulin-like growth factor-I and glucose-suppressed growth hormone levels after pituitary surgery in patients with acromegaly. Journal of Neurosurgery 200297 287-292.

30 Losa M, Gioia L, Picozzi P, Franzin A, Valle M, Giovanelli M \& Mortini P. The role of stereotactic radiotherapy in patients with growth hormone-secreting pituitary adenoma. Journal of Clinical Endocrinology and Metabolism 200893 2546-2552.

31 Aghi M \& Blevins LS Jr. Recent advances in the treatment of acromegaly. Current Opinion in Endocrinology, Diabetes and Obesity 200916 304-307.

32 Ronchi CL, Attanasio R, Verrua E, Cozzi R, Ferrante E, Loli P, Montefusco L, Motti E, Ferrari DI, Giugni E, Beck-Peccoz P \& Arosio M. Efficacy and tolerability of gamma knife radiosurgery in acromegaly: a 10 year follow-up study. Clinical Endocrinology 2009 $71846-852$.

33 Pollock BE. Stereotactic radiosurgery of benign intracranial tumors. Journal of Neuro-Oncology 200992 337-343.

34 Pollock BE, Brown PD, Nippoldt TB \& Young WF. Pituitary tumor type affects the chance of biochemical remission after radiosurgery of hormone-secreting pituitary adenomas. Neurosurgery $2008 \mathbf{6 2}$ 1271-1278.

35 Vik-Mo EO, Øksnes M, Pedersen P-H, Wentzel-Larsen T, Rødahl E, Thorsen F, Schreiner T, Aanderud S \& Lund-Johansen M. Gamma knife stereotactic radiosurgery for acromegaly. European Journal of Endocrinology 2007157 255-263.

36 Petit JH, Biller BMK, Coen JJ, Swearingen B, Ancukiewicz M, Bussiere M, Chapman P, Klibanski A \& Loeffler JS. Proton stereotactic radiosurgery in management of persistent acromegaly. Endocrine Practice 200713 726-734.

37 Sword FM, Allan CA, Plowman PN, Sibtain A, Evanson J, Chew SL, Grossman AB, Besser GM \& Monson JP. Stereotactic radiosurgery XVI: a treatment for previously irradiated pituitary adenomas. Journal of Clinical Endocrinology and Metabolism $2007 \mathbf{8 8}$ 5334-5340. 
38 Darzy KH \& Shalet SM. Hypopituitarism following radiotherapy. Pituitary 200912 40-50.

39 Mondok À, Szeifert GT, Mayer À, Czirják S, Gláz E, Nyáry I \& Rácz K. Treatment of pituitary tumours: radiation. Endocrine 2005 28 77-85.

40 Castinetti F, Nagai M, Morange I, Dufour H, Caron P, Chanson P, Cortet-Rudelli C, Kuhn JM, Conte-Devolx B, Regis J \& Brue T. Long-term results of stereotactic radiosurgery in secretory pituitary adenomas. Journal of Clinical Endocrinology and Metabolism 200994 3400-3407.
41 Ayuk J \& Stewart PM. Mortality following pituitary radiotherapy. Pituitary 200912 35-39.

42 Landolt AM, Haller D, Lomax N, Scheib S, Schubiger O, Siegfried J $\&$ Wellis G. Octreotide may act as a radioprotective agent in acromegaly. Journal of Clinical Endocrinology and Metabolism 2000 85 1287-1289.

Received 28 January 2010

Accepted 3 February 2010 